\title{
RLBM model: modeling of Manet's routing protocols based on restoration links break
}

\author{
Zahra Abdoly ${ }^{1}$, Seyed Javad Mirabedini ${ }^{2}$, Peyman Arebi ${ }^{3 *}$ \\ ${ }^{I}$ Department of Computer Science and Research branch, Islamic Azad University, Bushehr, Iran \\ ${ }^{2}$ Islamic Azad University Central Tehran Branch, Tehran, Iran \\ ${ }^{3}$ Technical and Vocational University, College of Bushehr, Bushehr,Iran \\ *Corresponding author E-mail: peymanarebi@gmail.com
}

Copyright () 2014 Arebi et al. This is an open access article distributed under the Creative Commons Attribution License, which permits unrestricted use, distribution, and reproduction in any medium, provided the original work is properly cited.

\begin{abstract}
Mobile ad hoc network is a temporary network that consists of a set of mobile nodes with wireless communication. There are several problems in the relationship between the components of these networks. Some of these problems are related to the routing problem. The main challenge in routing protocols of mobile ad hoc networks is links break phenomenon. This phenomenon has many negative impacts on the performance of routing protocols. In this paper, we attempted to study 10 routing protocols in mobile ad hoc networks that try to improve the performance of standard protocols of this type of network using the Restoration Links Break. By studying the behavior of these protocols, a common model used by all these protocols was introduced as Restoration Links Break model. Then the performance of each of the protocols was described based on the proposed model. On the other hand, we divided them into two categories according to protocol functions in Restoration Links Break. First category provides alternate routes before the Links Break event and the second category performs the replacement of route after the links break. Finally, the simulation of results revealed that the first category of protocols has a better delivery rate than the second category but the routing overhead of the second category is less than the first category.
\end{abstract}

Keywords: Mobile ADHOC Network, Routing Protocols, Restoration Links Break, Transmission.

\section{Introduction}

Mobile ad hoc networks is a self-organizing wireless networks for mobile device which is composed by a group of mobile terminals with wireless transceiver when the communication facility damaged between the mutual connections of sub elements [1]. Conflict in the accident of natural disaster such as non-center distributed controlled network may provide the temporary communication support compared with other communications networks, ad hoc networks has the following characteristics. Network is a self-regulating topology which is dynamic, bandwidth is restricted, and the capacity of network is changeable [1]. MANET is mobility where all the nodes are allowed to move in different dimensions which results in dynamic topology, since nodes are moving so they can go out of range network or come in range of network at any time, any node which part of network (n1) time $\left(\mathrm{t}_{\mathrm{j}}\right)$ can be part of an other network (n2) at time $\left(t_{j}\right)$ MANET are also used for meetings or other conventions in which people can quickly share information and data acquisition operations in hospitable terrain. The terminal energy is limited and the networks are controlled through distributing it and so on. The routing protocol in ad hoc network is critical in achieving good performance of networks. In this paper, in section 2 we introduce the important standard protocols in mobile ad hoc networks, then express the link break phenomenon in section 3 and then model the performance of routing protocols that have been proposed based on the Restoration Links Break in a mobile ad hoc network in section 4. Finally, in Section 5 these protocols are compared based on different criteria. 


\section{Routing protocols in manet}

Traditional routing protocols applied to wired networks are not suitable for ad hoc mobile wireless networks. For example, distance vector (Bellman-Ford) $[1,2]$ and link state routing schemes suffer from slow convergence to topology changes and bandwidth waste in periodical routing table exchanges. Especially, the routing loop in distance vector routing is another serious problem. Therefore, various routing protocols are proposed for ad hoc mobile wireless networks to solve the problems of routing loop and provide fast convergence to topology change. Broadly speaking, they are table-driven (e.g., DSDV [5], CGSR [4], and WRP [4]) and on-demand (or source-initiated, e.g., AODV [7], DSR [8], TORA [9], ABR [26], SSR [4], LAR [4], ZHLS [14], ODQOS [4], and DBR [26]) routing protocols [4]. Table-driven routing protocols demand that each mobile node should have up-to-date routing information of all nodes in the network. To achieve this goal, a routing table is maintained within each node and broadcast network-wide when network topology changes. Moreover, the routing table has to be exchanged periodically by broadcasting to all nodes in the network to keep track of the newest messages even though the network topology is not changed. Each node has routing information about all nodes of the whole network though most of it is undesired.

However, on-demand routing protocols have a totally different approach [15]; they create routes only when needed. Having data for transmission, a source node initiates a route discovery procedure to find the destination node. Route maintenance procedure is triggered whenever a route has been discovered and is in progress until the route is no longer required. The control messages used in on-demand routing protocols record only the nodes on the route, not all nodes in the network. Without maintaining the whole network topology, only if the route is changed, do the protocols proceed to maintain the route.

\section{Link break in routing protocols}

Though there are so many benefits offered by ad doc mobile wireless networks, the main fragility of the networks is the problem of a broken link.

\subsection{Link break problem}

MANET supports multi hop routing where the nodes other than the source and the destination nodes also take part in packet forwarding from one end to the other end. This results in the energy consumption of the intermediate nodes even though they are not the actual sender or receiver of the data [19]. The available battery power of the nodes decides the life time of the node as well as the whole network. In the Mobile Ad Hoc Network when data can be transmitted from source to destination in wireless network then most of the time there was the Link Failure problem occurred because of Range, Congestion, Delay and Battery Backup Problem [19]. There are no of routing problem include in mobile ad hoc network but most efficient and useful routing protocol is Reactive or On Demand (AODV) Routing Protocol. These On Demand routing protocol need to determine the link break in ad hoc network.

Mobile ad hoc networking is a challenging task due to the frequent changes in network topology as well as the lack of wireless resources [14]. As a result, routing in such networks experiences link failure more often. Hence, it is essential that a routing protocol for an ad hoc network considers the reasons for link failure to improve the routing performance. Link failure stems from node mobility and lack of network resources both resides in wireless medium and in nodes. Therefore it is essential to capture the characteristics to identify the quality of nodes and hence the quality of links. To identify the quality of nodes and quality of network, various levels are power level, Buffer level, stability level.

\subsection{Detection of broken link in mobile ad hoc network}

The link break can be detected by using Hello interval messages, MAC Layer Feedback and passive acknowledgement [14].

a) AODV usually transmit the Hello Interval messages at regular interval with default rate like once per second. These Hello messages determine the link availability between the source and destination. This Procedure works on the wired network which suffers from loss of packets and change of topology.

b) AODV uses the MAC Layer Feedback to find out Link break to neighboring nodes. This method is used for find the broken link quickly. MAC layer feedback method are performed on the network layer declaring the transmission error if the packets are not transmitting to the next neighbor node.

c) DSR uses the passive acknowledgement method if the MAC layer feedback method is not available. In this method a node after the transmission of packet to the next node to hear the channel that the next node further transmit the packet to the next neighbor node or not. If it is not transmit the packet to next node that means there is the link break in the network. This process needs the WLAN network card that is energy expensive. So this approach is not applicable in wireless ad hoc network only hello messages and MAC layer feedback methods are applicable for detection of link break in mobile ad hoc network. 


\subsection{The influence of link breaks on routing protocols}

The two categories of routing protocols of ad hoc mobile wireless networks are table-driven routing protocols and ondemand routing protocols [16]. Table-driven routing protocols have to maintain up-to-date routing information of all links in the network. Therefore, if any link in the network breaks, this kind of protocol has to trigger an update. This update is broadcasted network-wide till each node receives it. On the contrary, on-demand routing protocols only focus on the route they transmit packets, not on the whole networks. Hence, these protocols only need to maintain the links on a transmission route, not all the links of a network.

\section{Proposed model}

Many routing protocols have been proposed to improve the efficiency of routing process in mobile ad hoc networks. Each of these protocols has tried to enhance the efficiency of routing from a particular perspective. As mentioned above, Links Break phenomenon is one of the most effective factors in reducing the efficiency of routing protocols performance. For example, AODV protocol that is one of the most widely used routing protocols in mobile ad hoc networks has the main disadvantage of the lack of appropriate solution facing the Links Break phenomenon.

With the study of Links Break-based protocols and review of their performance, we found that all of these protocols use common methods for process improvement or prevent the Links Break. In this section we have shown the performance of protocols as a formal model. The presented model can be observed in Figure 1.

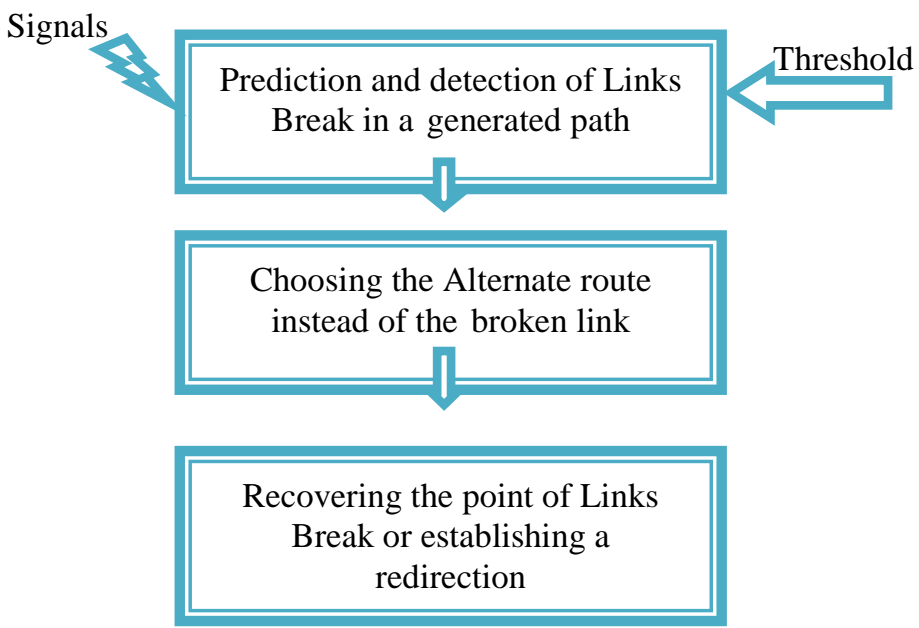

Fig. 1: The Structure of Links Break Model

As you can see in Figure 1, this model is composed of three distinct stages. Each protocol at first detects or predicts the Links Break phenomenon then chooses the best route to replace the broken link and finally repairs the new path from the point of break. In some cases, due to the lack of an alternative route, the situation is announced to the source node and it creates a new path from the source to the node.

The steps are implemented in each of the different routing protocols. Then, the performance of Links Break-based routing protocols is examined according to the each of the proposed steps of the model.

\subsection{Prediction and detection of links break step}

Whenever a protocol wants to mend a broken link, it must firstly diagnose the Links Break phenomenon in the network. In general, in order to increase the performance and maintain the route, Links Break is predicted in advance. This makes the protocol to find a way to repair the route before the loss of the current path and lost packet rates increase. The algorithm used in Prediction stage is shown in Figure 2 by protocols. The process used in Links Break prediction indicates that the protocols predict the occurrence of Links Break related to path by using criteria such as the signal strength of nodes on the path, distance between nodes, the energy levels of neighboring nodes, route traffic focus in a node and antenna nodes. In these protocols, a parameter called threshold is defined that is the base of detection and prediction of Links Break. The amount of valued criteria is compared with the threshold and if the values are inconsistent (in some protocols are more and in others less than the threshold value), informs its upstream node or in some protocols the source node. 


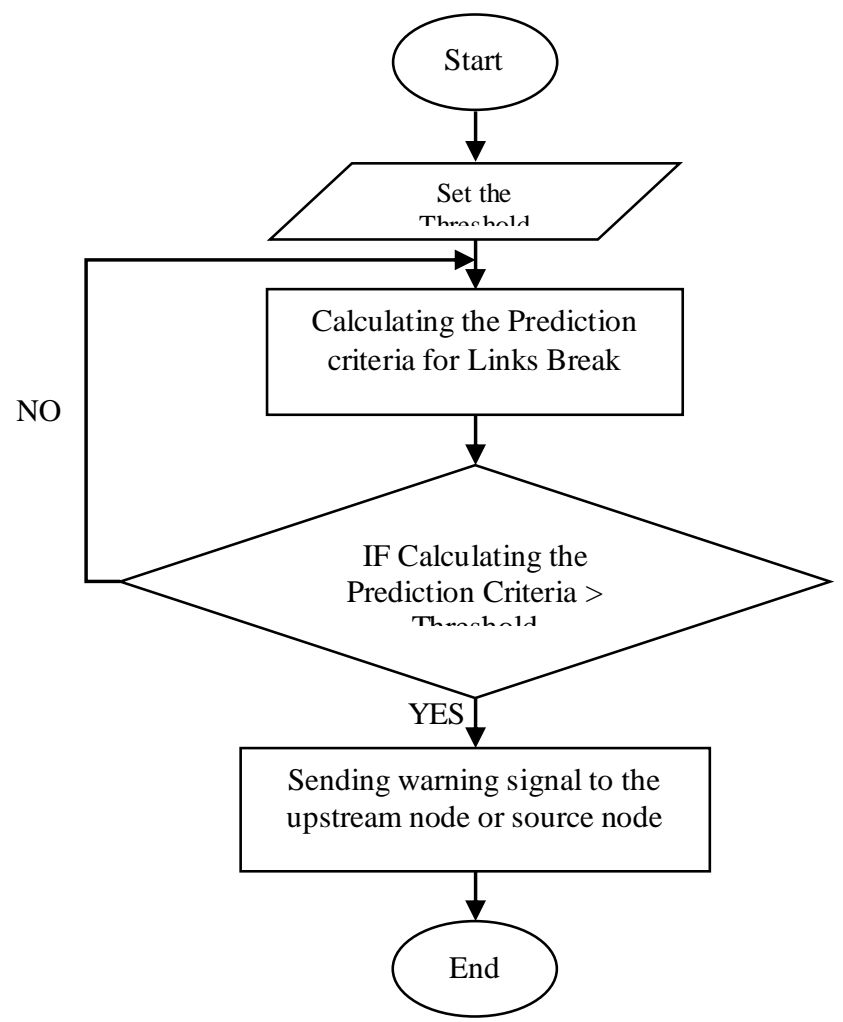

Fig. 2: The Algorithm Used By the Protocols to Detect the Links Break

ARM protocol [17] considers that the Links Break is based on the remove of node from the neighboring nodes area and the distance between nodes [4]. In this protocol, for each pair of nodes connected to each other, a common transmission range that corresponds to a radius between two nodes is calculated. For each node such as $\mathrm{p}$ to its neighbor such as $\mathrm{q}$ in the common transmission range $\mathrm{B}, \mathrm{TpB}$ value is calculated from the formula (1).

$\mathrm{T}_{\mathrm{pB}}\left(\mathrm{T}_{\mathrm{qB}}\right)=\operatorname{SQRT}\left(\left(\mathrm{R}_{2}-\left(\mathrm{x}_{\mathrm{pi}}-\mathrm{x}_{\mathrm{qi}}\right)\left(\mathrm{x}_{\mathrm{pi}}-\mathrm{x}_{\mathrm{qi}}+2\right)-\left(\mathrm{y}_{\mathrm{pi}}-\mathrm{y}_{\mathrm{qi}}\right)\left(\mathrm{y}_{\mathrm{pi}}-\mathrm{y}_{\mathrm{qi}}+2\right)\right) /(\mathrm{vp} \cos \theta-\mathrm{vq} \cos \alpha)^{2}+(\mathrm{vp} \sin \theta-\right.$ vqsin $\alpha 2$

By calculating the $\mathrm{T}_{\mathrm{pB}}$ value in equation (1), when the threshold is exceeded, the alarm signal will be sent.

In Protocols RAODV, AODV-BR, AODV-LR, AODV-ABR, AODV-ABL, in each node, a parameter as signal power of receiving node is calculated [29-33]. The protocols use the received signal strength or RSSA for calculating the value. In RSSA algorithms, equation (2) is used to calculate signal strength.

$p_{r}=\frac{P_{t} G_{t} G_{r} H_{t}^{2} H_{r}^{2}}{d^{4}}$

Two-Ray Ground Reflection model [29] used as the radio propagation model. $\mathrm{Pt}, \mathrm{G}_{\mathrm{t}}$ and $\mathrm{Ht}$ are the transmitted power, the antenna gain, and the height of the antenna on the transmitted side. $G_{r}$ and $\mathrm{Hr}$ are ones on the received side. $L$ is the loss factor of the system. In advance, each intermediate node transmits information of the transmitting side to the next hop of the destination route. The threshold of the received power which corresponds to the distance between nodes detecting the danger of the link break is defined from the above information. When the received power at the time of receiving data packets is less than the threshold and has decreased as compared with the previous received power, the node notifies the upstream node the danger of the link break. After that, the received RREQ which is transmitted from the upstream node is discarded and not processed for a while.

AODV-RD Protocol [32] diagnoses the distance between nodes such as AMR. This protocol uses equation (2) in other way for Links Break detection. In this protocol, distance variable (d) is calculated instead of the signal strength. In equation (2), if we want to calculate the variable $d$ this equation will become the equation (3).

$\mathrm{d}=\sqrt[4]{\frac{\mathrm{p}_{\mathrm{t}} \mathrm{G}_{\mathrm{t}} \mathrm{G}_{\mathrm{r}} \mathrm{H}_{\mathrm{t}}^{2} \mathrm{H}_{\mathrm{r}}^{2}}{\mathrm{p}_{\mathrm{r}}}}$

In this case, if the value of the parameter $d$ corresponding to each node is greater than the threshold value, the alarm signal will be sent. In mobile ad hoc network, nodes are moving at different speeds. On the other hand, nodes vary at different moments. AODV-LFP protocol [35] sends the warning signal using equation (3) and calculating the relative speed of each node with respect to its neighbors at any moment according to equation (4) and comparing it to the speed threshold. 
$\mathrm{V}=\frac{\sqrt[4]{\frac{\mathrm{p}_{\mathrm{t}} \mathrm{G}_{\mathrm{t}} \mathrm{G}_{\mathrm{r}} \mathrm{H}_{\mathrm{t}}^{2} \mathrm{H}_{\mathrm{r}}^{2}}{\mathrm{p}_{\mathrm{r} 2}}}-\sqrt[4]{\frac{\mathrm{p}_{\mathrm{t}} \mathrm{G}_{\mathrm{t}} \mathrm{G}_{\mathrm{r}} \mathrm{H}_{\mathrm{t}}^{2} \mathrm{H}_{\mathrm{r}}^{2}}{\mathrm{p}_{\mathrm{r} 1}}}}{\mathrm{t}_{1}-\mathrm{t}_{2}}$

PDSR protocol [36] defines a safe area for each node and calculates the received signal using the signal strength and the path loss rate according to equation 5 .

$\mathrm{P}_{\mathrm{r}}=\frac{\mathrm{P}_{0}}{\mathrm{r}^{\mathrm{n}}}$

Where $\mathrm{P} 0$ is the received signal strength, $\mathrm{r}$ is the safe area and $\mathrm{n}$ is the growth of lost paths. The value $\mathrm{n}$ is 2 to 4 . From Formula (5) the boundary of safe area is determined by taking the maximum value for the parameter $n$ (i.e. the worst case). Signal strength is calculated from the formula (6) at the boundary of the safe zone.

$\mathrm{P}_{\text {safe }}=\frac{\mathrm{P}_{0}}{\mathrm{~d}_{\text {safe }}^{4}}$

Where $r$ is equal to $d_{\text {safe }}$ and $n$ is equal to 4 (maximum value). Also $d_{\text {safe }}$ is equal to $d-w$ and $d$ is the distance between node to the neighbor and $\mathrm{w}$ is multiplied by the threshold value in relative velocity.

In AODV-BA protocol, for links break detection for each node, 4 parameters are calculated: radio reception, shared path, battery energy and density [19]. The radio reception is the signal strength of received node that is obtained from equation (2). Shared path means the number of active routes and that node is a part of it. The battery power of each node is calculated and is compared with a threshold value. Node density is the number of the node's neighbors that exchange information. The resultant of these four parameters is considered as the comparable value of the threshold.

\subsection{Choosing the alternative route step}

Upon receiving an alarm signal as the links break the protocols must take action to prevent the path break. In most of the standard protocols when faced with break, the source node is required to set aside the current route and establish a new route to the destination.

This imposes high costs on the network. Protocols reviewed in this paper have different functions. In these protocols, the action of alternative route occurs from the point of link break.

In terms of alternative route, discussed protocols can be divided into three categories. The first group is protocols that reserve the routes between two nodes as alternative routes before the links break and preserve the $s$ information about these routes in the internal tables of the node. This information is stored as part of the routing information. Also the request messages and alternative route response are used to create an alternative route like the messages that are exchanged in the communication path. These protocols include: AODV-BR, AODV-ABR, and PDSR. Figure 3 is an example of the establishment of alternative routes in AODV-BR protocol [30].

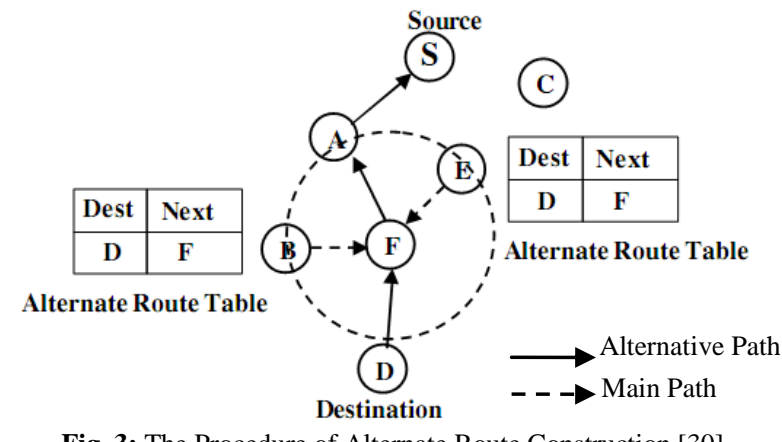

Fig. 3: The Procedure of Alternate Route Construction [30]

As you can see in Figure 3, the main route is established from the source node S. However, for node $\mathrm{F}$ alternative routes including its neighbors (B, E) are s selected when creating the route. In Nodes E and B, tables are maintained as an alternative route table which contains information of alternative routes. In this method, a node that is responsible for the repair chooses the best node in terms of space and bandwidth by referring to the tables of alternative routes related to the neighboring nodes.

The second group consists of protocols that are at the moment a node detects a link failure on the status of neighboring nodes connected to it, as an alternative route is selected. In this method, before any alternative route will not be created and maintained. Protocols, AMR, RAODV, AODV-LR, AODV-BA, and AODV-LFP are of these categories. Although all of these protocols, the decision to create an alternative route to link failures, but each performance is different. Failure that caused the link at ARM when compared to the distance between two nodes is diagnosed, intermediate nodes between two nodes that are intended as a bridge node is selected. The link between two nodes is divided into two communication links so that the distance will be maintained. In other protocols that are calculated based on the signal 
strength of nodes, node failure detector that has a lower cost is the link between your neighbors and the other node is connected to the other end of the broken link is selected as an alternate route.

Third group are protocols at different moments of the two methods before use. AODV-ABL protocols [30] of this category. In this protocol are the conditions that define the threshold value - the current state of the network routing is achieved at the moment - deciding that the first batch method is used to create an alternative path before the failure or the failure of the second group of methods is used. If the first method should be used in this protocol similar alternative route selection protocol, AODV-ABR and the second method is used to select alternative routes, such as protocols, AODV-LR.

\subsection{Route restoration}

After selecting the best route as an alternative route from the point of link failure, the alternate route protocols are part of the mainstream. The data in all tables of the nodes on the new path, and then send the updated data is used to transfer the new route. Note If the node detects a broken link, there is no alternative route, the current active route known as Route invalid error message is a node of origin. Then the source node will start again to establish a new route to the destination path.

\section{Comparison of links break protocols based on the proposed model}

The simulation of protocols [29-36] shows that all the results presented are based on the model of interpretation is based on repairing broken links. Simulations of these protocol standard protocols together with their families are compared. The main criteria for the calculation of all the protocols and routing protocols plays an important role in increasing efficiency, package delivery rate is the benchmark, the benchmark measure of routing overhead and average end-to-end delay. After reviewing the charts of vision simulation of standard delivery rate of packets, protocols, those protocols, the second group did better than the first. But the measure of the second category has lower overhead than the overhead of routing protocols are the first batch. From the perspective of the end-to-end delay measures the performance of both groups are close together but in most cases latter category better performance. In all protocols, with increasing numbers of nodes and connections in each of the three protocols has increased the efficiency criteria.

\section{Conclusion}

Link failure has devastating effects on the performance of routing protocols. Protocols proposed so far is that their main goal was to improve efficiency by repairing broken links. After reviewing these protocols, providing a common model that has been used for all these protocols, According to this model, the protocol in three steps: 1 -link failure prediction and detection, 2- alternative route, 3 - repair pathway, a link failure is encountered. From the perspective of an alternative route selection protocols can be divided into two categories the first category is an alternative process route before and concurrent with the creation of a link failure mainstream are doing, and the second after a failure to link this process to run. Compared to protocols in terms of performance based on the results of the simulations indicate that The first set of protocol standard delivery rates outperformed the control group and the second group performed better on the routing overhead.

\section{References}

[1] C.S. Murthy, B.S. Manoj, AdHoc Wireless Networks-Architecture and Protocols, Prentice Hall, (2004), pp: 336-338 and 627.

[2] R. E. Bellman, Dynamic Programming, Princeton University Press, Princeton 1957.

[3] T. Clausen, and P. Jacquet, "Optimized Link State Routing Protocol (OLSR)", IETF RFC3626. (2003), available online: http//www.ietf.org/rfc/rfc3626.txt.

[4] E. M. Royer, and C. -K. Toh, "A Review of Current Routing Protocols for Ad Hoc Mobile Wireless Networks", Proceedings of IEEE Personal Communications,(1999), pp: 46-54.

[5] C. E. Perkins and P. Bhagwat, "Highly Dynamic Destination-Sequenced Distance-Vector Routing (DSDV) for Mobile Computers," Proceedings of the ACM SIGCOMM, (1994), pp: 234-244.

[6] M. Gerla, X. Hong, G. Pei, "Fisheye State Routing Protocol (FSR)", IETF Internet Draft, work in progress, draft-ietfmanet- fsr-03. Txt, July 2002.

[7] S.Murthy and J.J.Garcia-Luna-Aceves, "An Efficient Routing Protocol for Wireless Networks", ACM Mobile Networks and Applications Journal", Special Issue on Routing in Mobile Communication Networks, Vol.1, No.2, (1996), pp.183-197. http://dx.doi.org/10.1007/BF01193336.

[8] C. Perkins, E. Belding-Royer, S. Das, "AODV Routing", RFC3561, Network Working Group, (2003).

[9] D. Johnson, B.D.A. Maltz, and Y.C.Hu, "The Dynamic Source Routing Protocol for Mobile Ad Hoc Networks (DSR)", draft-ietf-manet-dsr10.txt, (2004).

[10] V. Park, "Temporally-Ordered Routing Algorithm (TORA) Version 1 Functional Specification", (2001), available online: http://www.ietf.org/internet-drafts/draft-ietf-manet-tora-spec-04.txt.

[11] V. Park and M. Corson, "Highly Adaptive Distributed Routing Algorithm for Mobile Wireless Networks", Proceedings of 26th Annual IEEE INFOCOM 1997 Conference, Kobe, Japan, (1997), pp.1405-1413 
[12] Vidya Shree.P and Sophia Reena.G, "A Survey of Various Routing Protocols In Mobile Ad-Hoc Networks", International Journal of Computer Science and Engineering Technology, Vol. 3, No. 7, (2012). pp: 224-228.

[13] Zahedi Khalid, Ismail Abdul Samad,"Route Maintenance Approach for Link Breakage Prediction in Mobile Ad hoc Networks", International Journal of Advanced Computer Science \& Applications, Vol 2, No.10, (2011), pp: 23-30.

[14] Reena Rani, Reena Thakral, "Review On Mobile AD Hoc Network", Journal of Global Research in Computer Science, Volume 4, No. 4, (2013). Pp: 133-138.

[15] Vincent Harish, Vincent Shweta, "A Survey on Routing Protocols in Mobile Ad Hoc Networks", International Journal of Computer Science and Management Research, Vol. 1, No. 5, (2012).

[16] Ying-Kwei Ho and Wei Chang, " The Influence of Link Break on Wireless Ad Hoc Networks", Proceedings of International Symposium on Parallel and Distributed Processing with Applications, (2008), pp: 175-180

[17] Park Seungjin, Brian VanVoorst, "Anticipated Route Maintenance (ARM) in Location-Aided Mobile Ad Hoc Networks", Journal of Communications and Networks, Vol.7, NO.3, (2005), pp: 325-336. http://dx.doi.org/10.1109/JCN.2005.6389817.

[18] Sunil Taneja and Ashwani Kush, "A Survey of Routing Protocols in Mobile Ad Hoc Networks", International Journal of Innovation, Management and Technology, Vol.1, No. 3, (2010), pp: 279-285

[19] Utkarsh, Mukesh Mishra and Suchismita Chinar, "An Energy Saving Ad Hoc Routing Algorithm for MANET", Proceedings of IEEE- Fourth International Conference on Advanced Computing, Anna University, Chennai, (2012),PP:1-5

[20] Nazari, V and Ziyarati, K, performance Comparision of Routing Protocol for Mobile Ad Hoc Networks," Proceedings of IEEE Conference on Communications,Busan Korea, (2006), pp.1-5

[21] Arebi Peyman, A New Method for Restoration Link Break in Wireless Ad-hoc Networks by Estimation Energy Consumption, Proceedings of Computational Intelligence, Communication Systems and Networks, Phuket, Thailand, (2012), pp:377 - 381

[22] Ravindra.E1, VinayaDatt V Kohir2 and V. D Mytri3,"A Local Route Repair Algorithm Based On Link Failure Prediction in Mobile AdHoc Network", World Journal of Science and Technology, Vol.1, No.8, (2011), pp: 64-67.

[23] Jaing, S.M., He, D.J., Rao, J.Q., "A Prediction-Based Link Availability Estimation for Routing Metrices in MANETs", Proceedings of IEEE/ACM Transaction on Networking, Vol.13, No.6, (2005), pp: 1302-1312.

[24] Sharmila Sankar, Sankarana rayanan "A Cross Layer Design For Route Maintenance In Dense Manets", International Journal of Information Technology and Knowledge Management, Vol 4, No.2, (2011), pp 539-543.

[25] D.Nitnaware, A.Verma,"Energy Based Gossip RoutingAlgorithm for MANETs", Proceedings of International Conference on RecentTrends in Information, Telecommunication and computing, Kochi, Kerala, (2010), pp: 23 - 27.

[26] Y. Liang, S.F. Midkiff, "Multipath fresnel zone routing for wireless ad hoc networks", in: Proceedings of 2005 IEEE Wireless Communications and Networking Conference, vol.4, (2005), pp. 1958-1963. http://dx.doi.org/10.1109/WCNC.2005.1424819.

[27] Navid Nikaein and Christian Bonnet, "Improving Routing and Network Performance in Mobile Ad Hoc Networks Using Quality of Nodes", Modeling and Optimization in Mobile, Ad Hoc and Wireless Networks Journal, Vol.1, No.1, (2003).

[28] S.Perk and B.Voorst,"Anticipated Route Maintenance (ARM) in Location-Aided obile Ad-hoc Networks", Proceedings of Global Telecommunications Conference, Vol.5, NO.1, (2001), pp: 2809 - 2813.

[29] R.Senthil Kumar, P.Kamalakkannan, "Personalized RAODV algorithm for reduce link break in Mobile Ad Hoc Network", Proceedings of IEEE- Fourth International Conference on Advanced Computing, (2012), pp: 1-6.

[30] S.J.Lee and M.Gerla. "AODV-BR:Backup routing in Ad Hoc networks", Proceedings of IEEE wireless communication and Networking conference(WCNC), vol 3, (2000), pp:1316.

[31] Wei Kuang Lai, Sheng-Yu Hsiao, Yuh-Chung Lin: "Adaptive backup routing for ad-hoc networks". Computer Communications Journal, Vol.30, NO.2 (2007), pp: 453-464. http://dx.doi.org/10.1016/j.comcom.2006.09.011.

[32] LIU Jian, LI Fang Min, "An Improvement of AODV Protocol Based on Reliable Delivery in Mobile Ad hoc Networks", Proceedings of Fifth International Conference on Information Assurance and Security, VOL.1, (2009), pp: 507 - 510.

[33] Ali Khosrozadeh, Abolfazle Akbari, Maryam Bagheri, Neda Beikmahdavi," A New Algorithm AODV Routing Protocol in Mobile ADHOC Networks", International Journal of Latest Trends Computing, Vol-2 No 3,(2011),pp:457-464.

[34] Dimitri Marandin "Performance Evaluation of Failed Link Detection in Mobile Ad Hoc Networks", International Journal of Computer Applications, Vol.42, NO.2, (2012), pp: 398-404

[35] Qing Li,Cong Liu, Han-hong Jiang,"The Routing Protocol of AODV Based on Link Failure Prediction", Proceedings of 9th International Conference on Signal Processing, (2008), pp: 1993 - 1996

[36] Ramesh V., Subbaiah P., "Modified DSR(Preemptive) to reduce link breakage and routing overhead for MANET using Proactive Route Maintenance(PRM)", Global Journal of Computer Science and Technology, Vol 9,NO.5, (2010), pp:124-129. 